\title{
Rationalising dosage regimens of antimicrobial drugs: a pharmacological perspective
}

In recent years there has been much necessary debate over the increasing incidence of antimicrobial resistance to therapeutic agents and the consequent threat to their effectiveness in treating life-threatening diseases in human and veterinary medicine $[1,2]$. This has led many authorities to question current therapeutic strategies and practices. In 1998 concern within the European Community led to the Copenhagen recommendation $[1,3]$, actually a series of recommendations, the fifth of which stated that 'the European Union and member states should encourage the adoption of a wide range of measures to promote prudent use of antimicrobial agents'. To some authorities 'prudent' use might primarily or solely imply limitation on the extent of usage of antimicrobial drugs in human and veterinary medicine, for example through a general restriction of drug usage, or to restriction of individual drugs (or certain drug classes) for the treatment of lifethreatening infections. For example, the Food and Drug Administration (FDA) in the USA has recommended that consideration be given to restricting or prohibiting the use of fluoroquinolones in veterinary medicine [4].

While the prudent use of antimicrobial drugs is welcome and necessary, it is not sufficient. There is an additional urgent need to combine relevant pharmacological data with microbiological data to create new scientific approaches to the design of dosing schedules. This will ensure the rational use of antimicrobial drugs. Over several years there has been increasing recognition that the criteria adopted to design dosage schedules for clinical use have not provided schedules that are optimal. On the other hand, many proposals for developing new criteria have been made, but they have not yet had major impact on the antimicrobial resistance debate.

Pharmacology is the science of drug action, either directly on the body or on micro-organisms in or on the body (pharmacodynamics), together with the science of drug absorption into and fate within the body (pharmacokinetics). For antimicrobial drugs, it has been common to relate an easily determined in-vitro index of antimicrobial action, such as the minimum inhibitory concentration (MIC) or minimum bactericidal concentration (MBC), to pharmacokinetic data generated in vivo. The usual pharmacodynamic approach has been to determine the MIC against many isolates of a given species and, because sensitivity between strains may vary widely, the selected parameter has been the MIC for $50 \%$ or $90 \%$ of strains: MIC50 and MIC90, respectively. Pharmacokinetic approaches have generally involved establishing plasma concentration-time relationships and from these data variables such as $\mathrm{C}_{\max }, \mathrm{T}_{\max }$, area under plasma concentration-time curve (AUC), clearance, etc., have been determined. Even though the scientific literature describes more sophisticated or complex approaches, registration bodies responsible for licensing new medicinal products have generally provided guidelines that link a simple in-vitro pharmacodynamic variable to pharmacokinetic variables based on plasma concentration. For example, in the mid-1980s the body responsible for advising on the licensing of products for therapeutic use in animals, the Veterinary Products Committee, established two pharmacokinetic-pharmacodynamic criteria for setting dosage schedules: plasma concentration should be maintained in excess of $1 \times$ MIC90 for all pathogens against which activity is to be claimed for half the inter-dose interval; and plasma $\mathrm{C}_{\max }$ should be at least twice the MIC90 of the least sensitive micro-organisms. With hindsight, a number of comments might be made on these wellintentioned guidelines. First, conditions obtaining in broth culture measurements of MIC will not mimic precisely conditions occurring in body fluids in vivo. Moreover, no account is taken of such pharmacokinetic variables as degree of drug binding to plasma proteins and the fact that free drug concentration at the site of action may differ from both total and free concentrations in plasma. The time course of free drug concentration at the site of bacterial infection (which might be in the circulation, in interstitial fluid, or in transcellular fluids such as urine, etc.) may differ significantly from plasma concentration of total drug. In addition, a few drugs are converted to metabolites that may possess significant antimicrobial activity. Then there are the host defence mechanisms that operate in vivo but not under in-vitro conditions.

It must also be recognised that the two criteria do not take account of the mechanisms and types of action of antimicrobial drugs. To treat all drugs alike inevitably involves oversimplication of the complex conditions pertaining in vivo in patients (or animals) with infection. In clinically attainable concentrations, anti- 
microbial drugs may be classified into those with predominantly bacteristatic action and those that are primarily bactericidal; the latter may be further subdivided, so that in all there are three groups. Bacteristatic agents inhibit the growth of bacteria, so that clinical and bacteriological cure of infection is dependent on the integrity of host defences. Bactericidal drugs may be classified into agents that act by a concentration-dependent mechanism and those acting by a concentration-independent or time-dependent mechanism [4]. However, the distinction between concentration and time-dependent mechanisms of action is not absolute.

The last decade has witnessed evidence from both experimental and clinical studies to indicate that only limited benefit may be obtained with time-dependent bactericidal and bacteristatic drugs by achieving drug concentrations significantly exceeding MIC values. Several studies indicate that the period for which the concentration exceeds the MIC is the most important determinant of the outcome of therapy. When drug concentrations fall below the MIC, re-growth of the organism is likely. Therefore, some authors recommend that the drug concentration should remain above the MIC for the whole and not merely for some proportion of the inter-dose interval until clinical and bacteriological cure is effected. However, not all evidence indicates the necessity of maintaining plasma concentration in excess of the MIC for the whole of the interdose interval. For example, in otitis media in children bacteriological cure was of the order of $80-85 \%$ when concentrations of $\beta$-lactam antibiotics (time-dependent killing), co-trimoxazole (time-dependent killing) or macrolides (bacteristasis) were maintained above the MIC90 for $40-50 \%$ of the inter-dose interval [5]. The percentage cure achieved was little improved when the time in excess of the MIC was further increased - even to $100 \%$ of the inter-dose interval. Similarly, in a murine pneumonia model, based on Klebsiella pneumoniae, the largest reduction in $\log _{10}$ cfu was achieved when the time above the MIC was $40 \%$ of the dosage interval, although in this case a further modest reduction was achieved as the time above the MIC was increased to $100 \%$ [6]. In the light of these data, maintaining the time above the MIC for at least half the inter-dose interval would seem to be absolutely essential for bacteristatic and bactericidal agents that act mainly by a time-dependent killing mechanism, and perhaps it is desirable to achieve this for the whole of the interval wherever possible. This would seem sensible, given in-vitro evidence about the re-growth of organisms (sometimes variants exhibiting reduced susceptibility) when drug concentration falls below the MIC.

Antimicrobial drugs such as aminoglycosides and fluoroquinolones, the action of which is concentration-dependent, often exert a significant post-antibiotic effect and a post-antibiotic sub-MIC effect on suscep- tible micro-organisms. Inhibition of bacterial growth continues for a short time (occasionally for a few hours) after their complete removal. Studies in disease models have established that optimal outcome of therapy with these drugs is dependent on attaining high peak concentrations. The success of therapy with these agents correlates with the plasma AUC:MIC (AUIC) ratio, and avoidance of the development of resistance is dependent on the $\mathrm{C}_{\max }$ :MIC ratio. Thus, for fluoroquinolones and aminoglycosides $\mathrm{C}_{\max }$ :MIC ratios $>2$ should be obtained as recommended in the Veterinary Products Committee guidelines. Indeed, for fluoroquinolones, values of $\geqslant 10$ may be required for an optimal effect and AUIC ratios $>100$, and in some cases 250, have been recommended. Moreover, for aminoglycosides it has been shown that, while optimal antimicrobial effects correlate well with AUIC and the $\mathrm{C}_{\max }$ : $\mathrm{MIC}$ ratio, drug accumulation in the renal cortex (and nephrotoxicity) are less with pulse dosing than with continuous intravenous infusion. Hence, high doses given intermittently optimise efficacy and minimise toxicity $[7,8]$.

Investigations in our laboratory have established pharmacokinetic and pharmacodynamic properties of the fluoroquinolone antibacterial drugs, danofloxacin and marbofloxacin, in ruminant species [9-11]. To generate acute inflammatory exudate a tissue cage model of sterile inflammation was used, with intracaveal administration of the mild irritant carrageenan. Tissue cages in which carrageenan was not administered were used to harvest non-inflamed tissue cage fluid (transudate). Antibacterial activity of samples of each fluid, harvested at pre-determined times from calves, sheep and goats, against a clinical isolate of Mannheimia (Pasteurella) haemolytica was determined over a 24-h incubation period. Integration of the pharmacokinetic and pharmacodynamic data was undertaken by application of the classical sigmoidal $\mathrm{E}_{\max }$ equation. This approach has provided AUIC values for danofloxacin in serum, exudate and transudate that produce bacteristasis, bactericidal action (thousand-fold reduction in bacterial count) and elimination of organisms (reduction of the bacterial count to $\leqslant 10$ $\mathrm{cfu} / \mathrm{ml})$. These values relate to a single strain of one pathogen, and a next step is to use MIC90 values to determine AUIC values. The intention is to predict therapeutic dosages that can be expected to eliminate most organisms and not merely inhibit their growth or kill $99.9 \%$ of the infecting organism (bactericidal action). Of course, if this involves a significant increase in dosage of a given drug from that currently recommended, the possibility of increased toxicity will have to be considered.

Each of the parameters, time above the MIC, $\mathrm{C}_{\max }$ :MIC, and AUIC, relates a pharmacodynamic variable to a pharmacokinetic one. In patients (or animals) with clinical disease, both pharmacokinetic and pharmacodynamic components will vary (the 
former between individuals and the latter between strains and species of bacteria), sometimes within wide ranges. Pharmacokinetic differences may be caused by a wide range of factors, including age, weight, disease severity, route of drug administration, product formulation and, for orally administered drugs, food intake. There will also be inter-species and inter-strain variation in MIC. The distribution of MICs for a given species may be normal, log normal or even bimodal. To allow for these pharmacokinetic and pharmacodynamic variations new strategies for setting dosage schedules are required. We propose that population pharmacokinetic data for antimicrobial drugs in patients should be obtained to quantify the inter-subject variation in drug pharmacokinetics $[12,13]$. Population pharmacokinetic data may then be integrated with pharmacodynamic parameters such as MIC, bacteriological cure and clinical outcome. This approach requires appropriate mathematical modelling and statistical analysis. By this means, it is proposed that improved strategies can be developed for the selection of antimicrobial drug dosage schedules that optimise efficacy and minimise the opportunity for resistance development.

\section{P. LEES and F. SHOJAEE ALIABADI*} Royal Veterinary College, Hawkshead Campus, North Mymms, Hatfield, Hertfordshire AL9 7TA and

* Iranian Veterinary Organisation,

Veterinary Diagnostic Center and Pharmaceutical and Biological Products Quality Control Laboratories, Pajohesh Boulevard, Km 14 ${ }^{\text {th }}$ Tehran-Karaj Highway, Tehran, Iran (e-mail: plees@rvc.ac.uk)

\section{References}

1. European Commission. Opinion of the Scientific Steering Committee on antimicrobial resistance. Directorate-General XXIV, Consumer Policy and Consumer Health Protection, May 1999.

2. Apley MD, Brown SA, Fedorka-Cray PJ et al. Role of veterinary therapeutics in bacterial resistance development: animal and public health perspectives. J Am Vet Med Assoc 1998; 212: 1209-1213.

3. Midtvedt T. The microbial threat. The Copenhagen recommendation. Microb Ecol Health Dis 1998; 10: 65-67.

4. US Food and Drug Administration. A proposed framework for evaluating and assuring the human safety of the microbial effects of antimicrobial new animal drugs intended for use in food-producing animals. Federal Register 1999; 64 FR 887.

5. Hyatt JM, McKinnon PS, Zimmer GS, Schentag JJ. The importance of pharmacokinetic/pharmacodynamic surrogate markers to outcome. Focus on antibacterial agents. Clin Pharmacokinet 1995; 28: 143-160.

6. Craig WA. Choosing an antibiotic on the basis of pharmacodynamics. Ear Nose Throat J 1998; 77 (6 Suppl): 7-12.

7. Kluwe WM, Hook JB. Analysis of gentamicin uptake by rat renal cortical slices. Toxicol Appl Pharmacol 1978; 45: 531-539.

8. Czock D, Giehl M. Aminoglycoside pharmacokinetics and dynamics: a nonlinear approach. Int $J$ Clin Pharmacol Ther 1995; 33: 537-539.

9. Shojaee AliAbadi F, Lees P. Pharmacodynamic and pharmacokinetic inter-relationships of antibacterial drugs. $J$ Vet Pharmacol Ther 1997; 20: 14-17.

10. Shojaee AliAbadi F, Lees P. Pharmacodynamics of marbofloxacin in calves: evaluation in a tissue cage model. $J$ Vet Pharmacol Ther 1997; 20: 48-49.

11. Shojaee AliAbadi F, Lees P. Danofloxacin pharmacokinetics and ex vivo bactericidal activity in calves. AVTRW Annual Congress Proceedings. 1996; p. 19.

12. Vozeh S, Steimer J-L, Rowland M et al. The use of population pharmacokinetics in drug development. Clin Pharmacokinet 1996; 30: 81-93.

13. Martin-Jimenez T, Riviere JE. Population pharmacokinetics in veterinary medicine: potential use for therapeutic drug monitoring and prediction of tissue residues. J Vet Pharmacol Ther 1997; 21: 167-189. 